\title{
Research on the New Generation of Storage Bus-SAS
}

\author{
Nan Yao ${ }^{1}$, Junqin Huang ${ }^{2}$ \\ ${ }^{1)}$ State Grid Nanyang Power Supply Company, Nanyang, Henan, China \\ ${ }^{2)}$ State Grid Pingdingshan Power Supply Company, Pingdingshan, Henan, China
}

\begin{abstract}
For more than twenty years, parallel SCSI technology has become one of the mainstream bus for enterprise server. With the parallel bus frequency increases, and the transmitted signal cycle time is shorter, the number of parallel data from the sending end to the receiving end synchronous has become a big problem. Serial technology completely break the frequency limit of parallel bus, serial SCSI---SAS combined with the SCSI and SATA emerge as the times require, become a hot research topic in the industry.
\end{abstract}

Keywords - SAS, SATA, compatible

\section{新一代存储总线 SAS 的研究}

\author{
姚楠 $^{1}$ 黄军琴 $^{2}$ \\ 1) 国网南阳供电公司，南阳，河南，中国 \\ 2) 国网平顶山供电公司，平顶山，河南，中国
}

摘 要 二十多年来, 并行 SCSI 技术成为企业服务器端主流总线之一。随着并行总线频率的提高, 传送信号的周期时间越来越短, 而多位并行的数据如何从发送端同步到达接收端成了一大难题。串行技术的出现彻底打破了并行总线的频率极限, 结合了 SCSI 和 SATA 的串行 SCSI---SAS 应运而生, 成为当今业界的研究热点。

关键词 SAS, SATA, 兼容

1. 前言

2001 年 11 月 26 日, Compaq、IBM、LSI Logic Maxtor 和 Seagate 宣布成立 Serial Attached SCSI(SAS)工作组, 目 标是将并行 SCSI 与 SATA 的优点相结合, 定义一个新型串 行点对点的企业级存储设备接口。2004 年 1 月 28 日, ANSI(美国国家标准协会)批准通过 SAS 标准。SAS 第一代 规范传输速率为 $3.0 \mathrm{~Gb} / \mathrm{s}$ 。SAS 作为 Ultra320 SCSI 接口 之后所开发出的全新接口, 吸取了 FC 的一些优点, 成为 企业级串行接口的理想选择。该接口的设计是为了改善存 储系统的效能、可用性和扩充性, 以及提供与 SATA 硬盘 的兼容性。SAS 与 SATA 为兼容的传输技术, 二者可同时 存在于一个存储系统中, 并满足各种广泛的需求。

\section{SAS 的基本结构}

SAS 起步于 SATA 之后。所以串行 SCSI 工作组在制定 SAS 标准时很自然就借鉴了成熟的串行 ATA 的成功经验和 技术, 并在最大程度上兼容 SATA 设备。SAS 的基本结构
与 SATA 十分相似。现主要从 SAS 物理连接、协议结构 和扩展器三大特点来讲述。

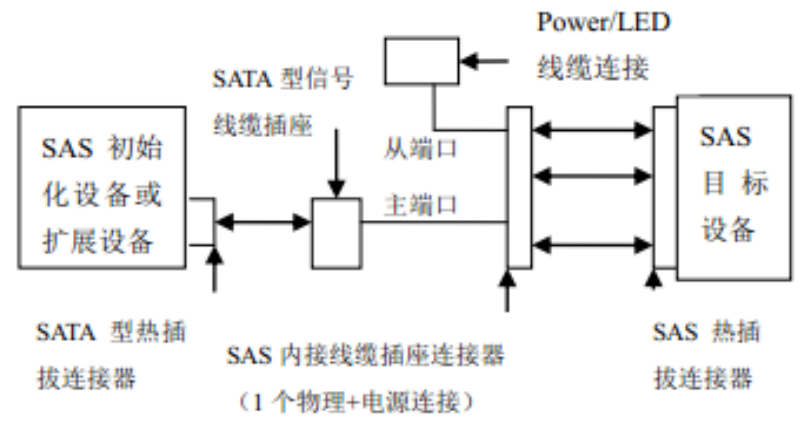

图 $1 \mathrm{SAS}$ 内部连接

\subsection{SAS 物理连接}

SAS 线缆和物理连接分为内部和外部两种。这也是相 对 SATA 的仅有内部连接不同的一大特点。 
图 1 中显示了单端口内接。内部连接器也支持 2 个物 理连接, 即双端口内接。将电源接口做在一起, 并采用了 增强的 SATA 物理层(添加第二端口支持)。

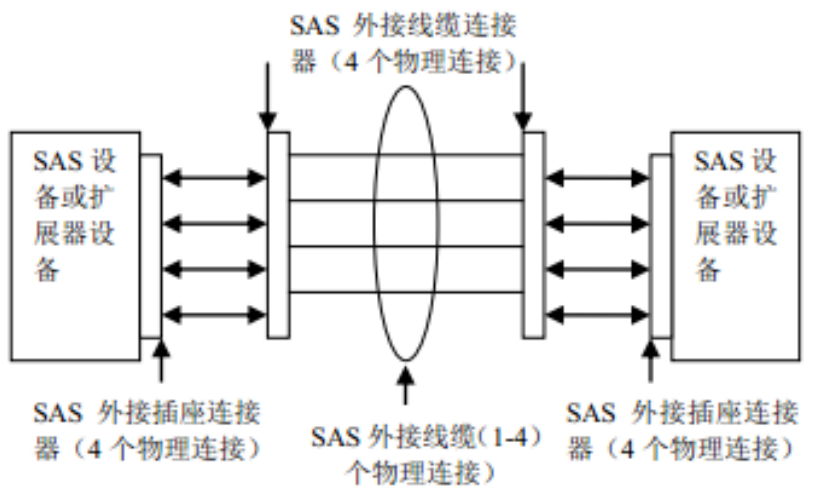

图 $2 \mathrm{SAS}$ 外部连接

图 2 中所示的是 SAS 的外部连接。在 SATA 中, 采用 的是半双工模式。SAS 采用的是全双工传输模式。一条串 行链路用于数据发送, 一条串行链路用于数据接收, 在一 个时钟周期内, 数据发送和接收工作可以同时完成。而在 每条串行链路中, 一条线路传输正信号, 表示 “1”; 一条 线路传输负信号，表示 “ 0 ”。这就是差分信号技术。所以 SAS 在物理上需要两个差分信号对和四条线路。

\subsection{SAS 协议结构}

SAS 标准层分为应用层、传输层、端口层、链路层、 Phy 层和物理层。应用层接收用户发送的数据操作请求; 传输层负责对数据进行组装和帧定义; 端口层判断数据所 属的是否为多端口系统; 链路层进行帧传输; 数据帧在 Phy 层以 $8 \mathrm{bit} / 10 \mathrm{bit}$ 格式进行编码; 由物理层传输数据包。

其中, SAS 的三个协议 SSP (Serial SCSI Protocol, 串 行 SCSI 协议)、STP (Serial ATA Tunneling Protocol, 串行 ATA 通道协议) 和 SMP (Serial Management Protocol, 串 行管理协议) 是 SAS 的另一大特点。SSP 负责串行 SCSI 相关的应用, 其主要部分直接继承了并行 SCSI 的命令集。 STP 负责处理串行 ATA 兼容的应用。SMP 负责对 Expander (扩展器) 的管理。有关扩展器的说明将在下一节中详细 讲述。

\subsection{SAS 扩展器}

在多 SAS 设备连接中, 扩展器设备担任了传输服务和 促进通信的角色。扩展器包含有至少 2 个外在扩展器端口
河至少一个用来管理的 SMP 目标端口。根据路由能力复杂 程度的不同, 扩展器可分为 Edge 扩展器和 Fan-out 扩展器。 每个 Edge 扩展器可连接的其他扩展器不能超过一个, 可连 接的 SAS 或 SATA 端口则可达 64 个, 如果拓扑结构需要 2 个以上的 Edge 扩展器, 就必须用 Fan-out 扩展器来提供并 管理增加的连接; 每个 SAS 域至多有一个 Fan-out 扩展器, 它能连接的 Edge 扩展器和端口不超过 64 个, 寻址能力最 高可达 4096(64X 64)台设备。

\section{SAS 与 SATA 的比较}

\subsection{SAS 与 SATA 的兼容}

SAS 和 SATA 之间兼容性给普通用户, 系统构建者和 系统管理员带来诸多的好处。SAS 和 SATA 之间兼容性又 分为物理层和协议层上的兼容。

SAS 连接接口和 SATA 接口完全兼容, SATA 硬盘可以 直接使用在 SAS 的环境中。从接口标准上而言, SATA 是 SAS 的一个子标准, 因此 SAS 控制器可以直接操控 SATA 硬盘。但是 SAS 却不能直接使用在 SATA 的环境中, 因为 SATA 控制器并不能对 SAS 硬盘进行控制。SAS 和 SATA 之间兼容性给普通用户, 系统构建者和系统管理员带来诸 多的好处。

SAS 由 3 种类型协议组成, 根据连接的不同设备使用 相应的协议进行数据传输。其中 SATA 通道协议 (STP) 用 于 SAS 和 SATA 之间数据的传输。

\section{$3.2 \mathrm{SAS}$ 与 SATA 的区别}

SAS 和 S-ATA 的区别源于他们各自的应用领域不同,

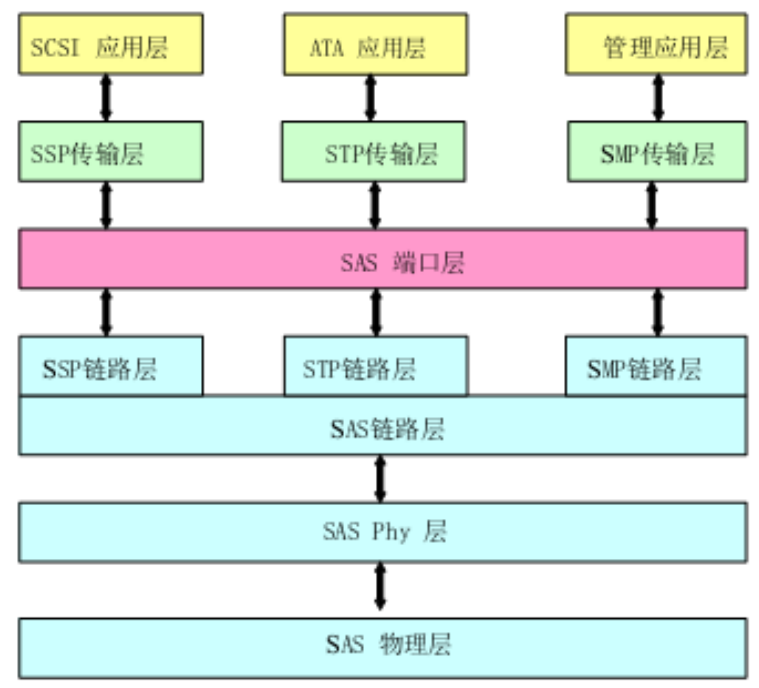

图 3 SAS 协议 
S-ATA 主要是针对 PC 桌面机及笔记本电脑。而 SAS 主要 是针对服务器和企业级存储开发的技术。另外, 二者的 RX 和 TX 差分电压的范围也不同, SAS 驱动信号的电压范围 要高于 S-ATA, 而且线缆的长度也更长, 可以长达 10 米。 在初始化阶段, 兼容 3 个 SAS 协议的 SAS 系统可识别连接 到各端口的设备类型, 并且调整与它匹配的差分电压驱动 该设备。

表 1 详细的描述了 SATA 与 SAS 的主要区别。

\section{表 1 SAS 与 SATA 比较}

\begin{tabular}{|l|l|l|}
\hline \multicolumn{1}{|c|}{ 规格 } & \multicolumn{1}{|c|}{ SATA1.0 } & \multicolumn{1}{c|}{ SAS } \\
\hline 设备/通道 & 1 & 128 \\
信号的传输速率 & $150 \mathrm{MB} / \mathrm{s}$ & $150 / 300 \mathrm{MB} / \mathrm{s}$ \\
Tx 电压 & $400-600 \mathrm{mV}$ & $800-1600 \mathrm{mV}$ \\
Rx 电压 & $325-600 \mathrm{mV}$ & $275-1600 \mathrm{mV}$ \\
双端口 & 无 & 有 \\
线缆最大长度 & $1 \mathrm{~m}$ & $10 \mathrm{~m}$ \\
应用范畴 & 桌面 PC & 工作站/企业 \\
\hline
\end{tabular}

SATA 与 SAS 的另一区别是物理连接器的不同: SAS 的连接器兼容于 S-ATA, 其中“s”代表信号针, 而“p”代表 电源针。SATA1.0 的 p11 是预留无定义的, 而 SAS 定义为 预备指示信号用, 提供 SAS 设备的活动指示灯; SAS 为能 使用双端口, 还定义了 S8-14 信号针; 为防止 S-ATA 设备 误插入 SAS 连接器, SAS 连接器加入了一个凹口。

\section{SAS 的应用和发展}

在市场应用方面, SAS 有望打破 SCSI 接口技术一直作 为企业级存储的首选。而 SAS 硬盘将使企业存储平台发生 巨大的改变。在 2002 年, 业界曾预测 SAN 和 NAS 等外部 网络存储设备是未来发展的趋势。一是存储系统价格的迅 速下滑等因素让 SAS 硬盘受到业界的一致关注。二是 SAS
系统和 SATA 系统的兼容性。SAS 和 S-ATA 类似的物理和 电气特征, 使得研发者有可能开发一种公用的 SAS 连接器 背板。

根据各厂商的发展计划。2004 年下半年就将会有 SAS 技术的存储系统上市。Maxtor 公司预计。到 2009 年将有三 分之二的外部存储设备采用 SAS 技术。以连接 SAS 或 SATA 硬盘。而厂商们也相继推出相应产品: 2004 年初, 希捷展出了 SAS 接口的硬盘驱动器 2.5 英寸万转专业硬盘 Savior 10K.1 和 3.5 英寸万转企业级 Cheetah 15K.4 。而 LSI 在推出 4 端口控制器 IC--LSISAS1064 后又继续批量生产 8 端口的控制器 IC。可以看到, SAS 技术背景强硬, 市场前 景巨大。

\section{5. 小结}

SAS 作为新一代的硬盘总线接口技术。SAS 的三个协 议 SSP 协议、STP 协议和 SMP 协议很好的继承了 SCSI 的 命令集, 也在最大程度上实现了对 SATA 的兼容。同时, SAS 的扩展器的路由性能使 SAS 系统中能够级联式的挂载 很多个 SAS 或 SATA 设备。我们可以看到, SAS 在服务器 和网络存储方面的应用必将成为企业级客户的首选。

\section{参考文献}

[1] Susan Bobholz. Serial Attached SCSI and Serial ATA Compatibility. www.Intel.com. 2002

[2] T10. Information technology-Serial Attached SCSI-1.1.T10. 2005-3

[3] Hua Zheng, Jinqi Gu. A profile of the new generation serial storage bus. High performance computing technology,2004-4

[4] Nan Xiao.SAS and SATA: Excellent compatibility. Netinfo Security, 204-10

[5] Guangbin Zhang. Specifications of Serial Attached SCSI. China Computer World, 2003-8 